\title{
A numerical approach to modelling avascular tumour evolution with white noise
}

\author{
Keng-Cheng Ang ${ }^{1} \quad$ Liang-Soon Tan ${ }^{2}$
}

(Received 22 July 2008; revised 4 December 2008)

\begin{abstract}
A model for avascular tumour growth with white noise is presented. The model is a set of partial differential equations describing the spatio-temporal evolutions of cell concentrations based on reactiondiffusion dynamics and the law of mass conservation. Perturbations in the form of white noise are introduced to model the effects of random processes on distinct time scales. Numerical simulations in one and two space dimensions are presented. Numerical results indicate that the proposed model is a reasonable approach that may be used to examine the effects of nutrient supply in tumour growth dynamics.
\end{abstract}

\section{Contents}

1 Introduction

C570

http://anziamj . austms.org.au/ojs/index.php/ANZIAMJ/article/view/1362 gives this article, (c) Austral. Mathematical Soc. 2008. Published December 17, 2008. ISSN 1446-8735. (Print two pages per sheet of paper.) 
2 Tumour growth model with white noise

3 Numerical solution

C573

4 Results and discussion

C576

5 Conclusion

C580

References

C581

\section{Introduction}

This article focusses on the growth dynamics of the avascular tumour. After the early stages of growth, a multicell spheroid typically consists of an inner zone of necrotic cells and an outer zone of living cells. The outer zone can be further divided into a layer of quiescent cells and a layer of proliferating cells. Quiescent cells are alive, but do not proliferate due to nutrient deprivation.

Figure 1 illustrates the development of a mass of tumour cells to the typical three layer structure of a multicell spheroid consisting of a thin outer shell of proliferating cells, an inner region where cells are dormant but viable, and a central region of necrotic material.

Modelling of avascular tumours are often seen as a first step towards developing more models for later stages of tumour growth. Burton [4], Adam [2] and Ward [13] overview the different types of mathematical models that have been developed over the years. More recent studies involving stochastic models include those developed by Albano and Giorno [3], as well as Lo [9].

In this article, white noise is introduced into a reaction-diffusion model for avascular tumour growth. A numerical approach then solves the set of governing model equations. A simulation of tumour growth in two dimensions is also presented to better visualize the growth process. 


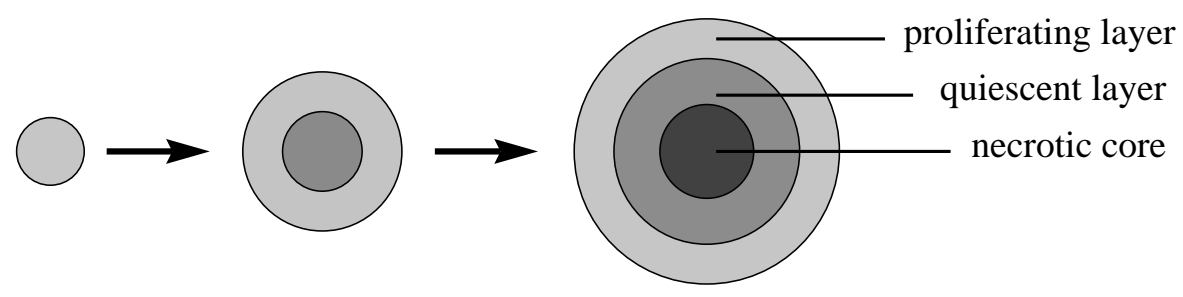

Figure 1: Schematic illustration showing the growth of a tumour and the formation of the proliferating and quiescent layers, and the necrotic core

\section{Tumour growth model with white noise}

The tumour model presented here is based on the compartment model shown in Figure 2. Originally proposed by Sheratt and Chaplain [11], the in vivo tumour is modelled as a continuum of proliferating, quiescent and necrotic cells, whose densities are denoted by $p(x, t), q(x, t)$ and $n(x, t)$ respectively, where $t$ and $x$ are time and the one dimensional space coordinate respectively.

The mitosis rate $\mathrm{g}(\mathrm{c})$ of the proliferating cells is assumed to be proportional to the concentration of nutrients and limited by the crowding of the total cell population. Nutrients are assumed to pass through the surface of the tumour and diffuse into the interior through the intracellular space fast enough for the local nutrient concentration $c(x, t)$ to be quasi-steady. In the direction of the core of the tumour, some proliferating cells become quiescent at rate $f(c)$, and some quiescent cells undergo necrosis at rate $h(c)$.

As described by Tan and Ang [12], assuming a one dimensional model, the set of equations governing the evolution of $p(x, t), q(x, t)$ and $n(x, t)$, and the equation for $c(x, t)$ are

$$
\frac{\partial p}{\partial t}=\frac{\partial}{\partial x}\left[\frac{p}{p+q} \frac{\partial(p+q)}{\partial x}\right]+g(c) p(1-p-q-n)-f(c) p
$$




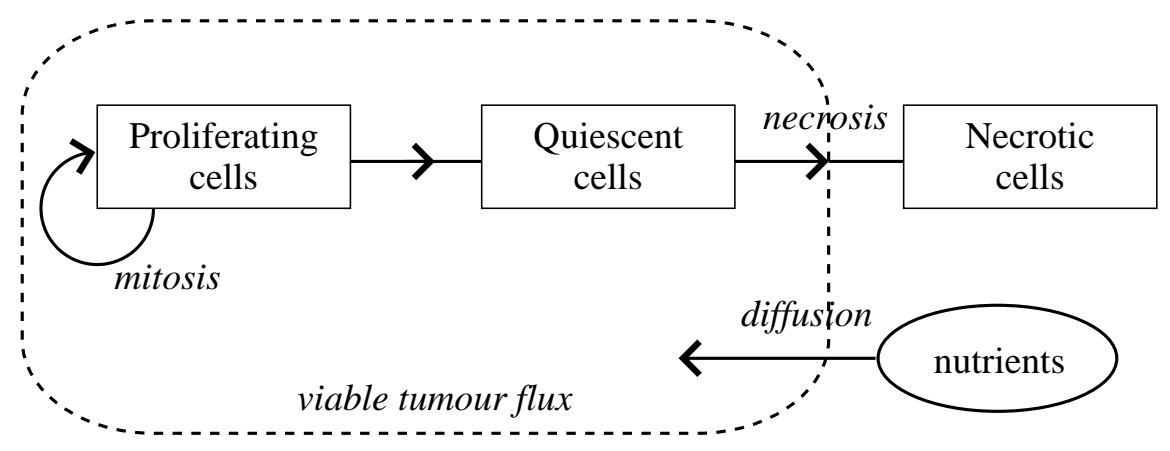

Figure 2: A compartment-model for tumour growth: proliferating cells may multiply through mitosis, or become quiescent cells, which in turn may undergo necrosis

$$
\begin{aligned}
\frac{\partial q}{\partial t} & =\frac{\partial}{\partial x}\left[\frac{q}{p+q} \frac{\partial(p+q)}{\partial x}\right]+f(c) p-h(c) q \\
\frac{\partial n}{\partial t} & =h(c) q \\
c & =\frac{c_{0} \gamma}{\gamma+p}[1-\alpha(p+q+n)] .
\end{aligned}
$$

Equation (4) represents the access of nutrient from underlying tissue. We assume that nutrient can only reach the viable tumour cells at the centre by diffusing through the whole tumour structure. By assuming that the effectiveness of this source term decreases with overall cell density, the parameter $\alpha \in(0,1]$ represents a constant of proportionality and $c_{0}$ is the nutrient concentration in the absence of a tumour cell population. The parameter $\gamma$ represents the ratio of the rates of nutrient supply to nutrient depletion as proposed in the original model developed by Sheratt and Chaplain [11].

The model equations have been rescaled in space by assuming that a nondimensionalised cell density of one corresponds to a completely close packed cell population. A suitable time rescaling is achieved by fixing $g(0)=1$. In 
subsequent numerical computations, at $t=0$ and $x=0$ the total tumour cell density $p+q+n$ is set to one for convenience. The functions $f(c)$ and $h(c)$ are assumed to be decreasing with c. A Gompertz growth rate is used to represent the mitosis term in the formulation of $\mathrm{g}(\mathrm{c})$.

In Equations (1) and (2), the movement terms of the proliferating and quiescent cells are represented respectively by

$$
\frac{\partial}{\partial x}\left[\frac{p}{p+q} \frac{\partial(p+q)}{\partial x}\right] \text { and } \frac{\partial}{\partial x}\left[\frac{q}{p+q} \frac{\partial(p+q)}{\partial x}\right] .
$$

These terms model the effects of contact inhibition of cell migration [1] by fractionating the overall cell flux evenly between the proliferating and quiescent cell densities.

Given the random effects due to the disparate cell clones, cell stress and inhibiting factors considered in this model, it is appropriate to inject a stochastic component to the model. One approach is to introduce white noise to the Equation (3), the necrosis equation. The result is the following equation in differential form,

$$
\partial \mathfrak{n}=h(c) q \partial t+q \tau d W(t),
$$

where $W(t)$ is the standard Wiener process and $\tau$ is a scaling parameter.

Equations (1), (2), (4) and (5), together with functions $\mathbf{f}(\mathbf{c}), \boldsymbol{g}(\mathbf{c})$ and $\boldsymbol{h}(\mathbf{c})$, form a set of equations that model the evolution of cells with white noise perturbations in an avascular tumour.

\section{Numerical solution}

The system of equations (1), (2), (4) and (5) are discretized using a forward difference approximation for time derivatives and central difference approxi- 
mations for space derivatives. The resulting set is

$$
\begin{aligned}
p_{i}^{j+1} & =p_{i}^{j}+\Delta t\left[u_{i}^{j}+g\left(c_{i}^{j}\right) p_{i}^{j}\left(1-p_{i}^{j}-q_{i}^{j}-n_{i}^{j}\right)-f\left(c_{i}^{j}\right) p_{i}^{j}\right], \\
q_{i}^{j+1} & =q_{i}^{j}+\Delta t\left[v_{i}^{j}+f\left(c_{i}^{j}\right) p_{i}^{j}-h\left(c_{i}^{j}\right) q_{i}^{j}\right], \\
n_{i}^{j+1} & =n_{i}^{j}+\Delta t\left[h\left(c_{i}^{j}\right) q_{i}^{j}\right]+\tau q_{i}^{j}\left(W_{i}^{j+1}-W_{i}^{j}\right), \\
c_{i}^{j} & =\frac{\gamma}{\gamma+p_{i}^{j}}\left[1-\alpha\left(p_{i}^{j}+q_{i}^{j}+n_{i}^{j}\right)\right]
\end{aligned}
$$

where

$$
\begin{aligned}
u_{i}^{j}= & {\left[\left(p_{i+1}^{j}-p_{i-1}^{j}\right) r_{i}^{j}\left(r_{i+1}^{j}-r_{i-1}^{j}\right)+4 p_{i}^{j} r_{i}^{j}\left(r_{i+1}^{j}-2 r_{i}^{j}+r_{i-1}^{j}\right)\right.} \\
& \left.-p_{i}^{j}\left(r_{i+1}^{j}-r_{i-1}^{j}\right)^{2}\right] /\left[4(\Delta x)^{2}\left(r_{i}^{j}\right)^{2}\right], \\
v_{i}^{j}= & {\left[\left(q_{i+1}^{j}-q_{i-1}^{j}\right) r_{i}^{j}\left(r_{i+1}^{j}-r_{i-1}^{j}\right)+4 q_{i}^{j} r_{i}^{j}\left(r_{i+1}^{j}-2 r_{i}^{j}+r_{i-1}^{j}\right)\right.} \\
& \left.-q_{i}^{j}\left(r_{i+1}^{j}-r_{i-1}^{j}\right)^{2}\right] /\left[4(\Delta x)^{2}\left(r_{i}^{j}\right)^{2}\right], \\
r_{i}^{j}= & p_{i}^{j}+q_{i}^{j},
\end{aligned}
$$

and $\Delta t$ and $\Delta x$ refer to the time steps and space intervals respectively for the finite difference scheme. In the above equations, the superscript and subscript represent the time level and space position respectively. In other words, $u_{i}^{j}$ denotes $u\left(x_{i}, t_{j}\right)=u(i \Delta x, j \Delta t)$. Tan and Ang [12] describe the implementation more completely.

The Euler-Maruyama (EM) method for solving stochastic differential equations numerically as suggested by Higham [8] is used here. A discretized Brownian path over $[0, T]$ with a prescribed incremental value of $\delta t$ is first computed. Equation (8) is then solved by applying the EM method, with a stepsize of $\Delta \mathrm{T}=\mathrm{R} \delta \mathrm{t}$, for some $\mathrm{R} \in \mathrm{Z}^{+}$. In other words, we always choose the stepsize for the numerical method, $\Delta T$, to be an integer multiple $R \geq 1$ of the increment $\delta \mathrm{t}$ for the Brownian path. This ensures that the set of points 
TABLE 1: Functional forms and parameter values used in present model.

\begin{tabular}{lc}
\hline Functions & Possible parameter values \\
\hline $\mathrm{f}(\mathrm{c})=\frac{1}{2}(1-\tanh (4 \mathrm{c}-2))$ & $\gamma=10, \mathrm{c}_{0}=1$ \\
$\mathrm{~g}(\mathrm{c})=\beta \mathrm{e}^{\beta \mathrm{c}}$ & $\beta=0.1$ to 1.0 \\
$\mathrm{~h}(\mathrm{c})=\frac{1}{2} \mathrm{f}(\mathrm{c})$ & $\alpha=0.2$ to 0.9 \\
\hline
\end{tabular}

on which the discretized Brownian path is based contains the points of the EM computation.

On a general step, the EM method requires the increment

$$
W_{i}^{j+1}-W_{i}^{j}=W((j+1) R \delta t)-W(j R \delta t)=\sum_{k=j R+1}^{(j+1) R} d W_{k},
$$

with $W(0)=0$.

For boundary conditions, we set $\partial p / \partial x=0$ and $\partial q / \partial x=0$ at $x=0$ and as $x \rightarrow \infty$. We assume that at $t=0$ there are no quiescent and necrotic cells. Thus, we set $q(x, 0)=\mathfrak{n}(x, 0)=0$. At the beginning of any tumour growth, there would be a high concentration of proliferating cells near the point of genesis. The density of proliferating cells decreases with distance from the point of genesis. Thus, it is assumed that $p(x, 0)$ takes the form of a decreasing exponential function.

For this discussion, $f(c)$ and $h(c)$ have been intentionally chosen to coincide with those used by Sheratt and Chaplain [11] and Tan and Ang [12] for comparison purpose, while $\mathrm{g}(\mathrm{c})$ is given a Gompertz growth formulation as discussed earlier. Similarly, values for various parameters in the model are chosen to be those used by these authors. These are shown in Table 1.

In the present study, we let $p(x, 0)=0.01 \exp (-0.1 x)$, and set $\delta t=0.05$ and $R=2$. In solving the finite difference equations, we let $\Delta t=0.004$ and $\Delta x=1$. For the current discussion, we set the duration of the simulation 
to be $\mathrm{T}=14$ (in arbitrary time units). As for the one dimensional space, theoretically the domain is $[0, \infty]$. However, in practice the numerical computations may terminate when $x$ is sufficiently large. Numerical experiments indicate that $x=210$ is deemed far enough if $\Delta x=1$.

The Box-Muller random number generator is used to generate a set of random numbers from a Normal distribution with mean zero and variance one. The code was written in Visual Basic for Applications (VBA) and compiled and run on a Pentium 4 system. Computation was stable for the chosen set of parameters and convergence was rapid.

\section{Results and discussion}

The model is solved for the set of parameters, functions, boundary and initial values mentioned above. Values of $\alpha$ ranging from 0.2 to 0.9 were used in the simulation runs to investigate the effects of variable nutrient supply on tumour growth dynamics. Results for representative cases $\alpha=0.4$ and $\alpha=0.8$, with $\beta=0.5$ are presented in Figures 3 and 4 .

The distributions of the tumour cell densities are found to be significantly different from the models originally proposed by Sheratt and Chaplain and later modified by Tan and Ang. In these earlier models, predicted cell distributions are either unrealistically smooth and symmetrical, or contain too many jumps and apparent discontinuities. In contrast, the current model shows a smooth but asymmetric spatial distribution of tumour cells.

From the figures, we observe that from $t=2$ to $t=8$, the initial necrotic cell density is distributed inwards of the tumour core. As time passes, the necrotic cells continue to internalize with some necrotic cells dispersing towards the outer edge of the tumour due to limited nutrient access.

As the necrotic cell density builds up, no tumour regression is detected; 

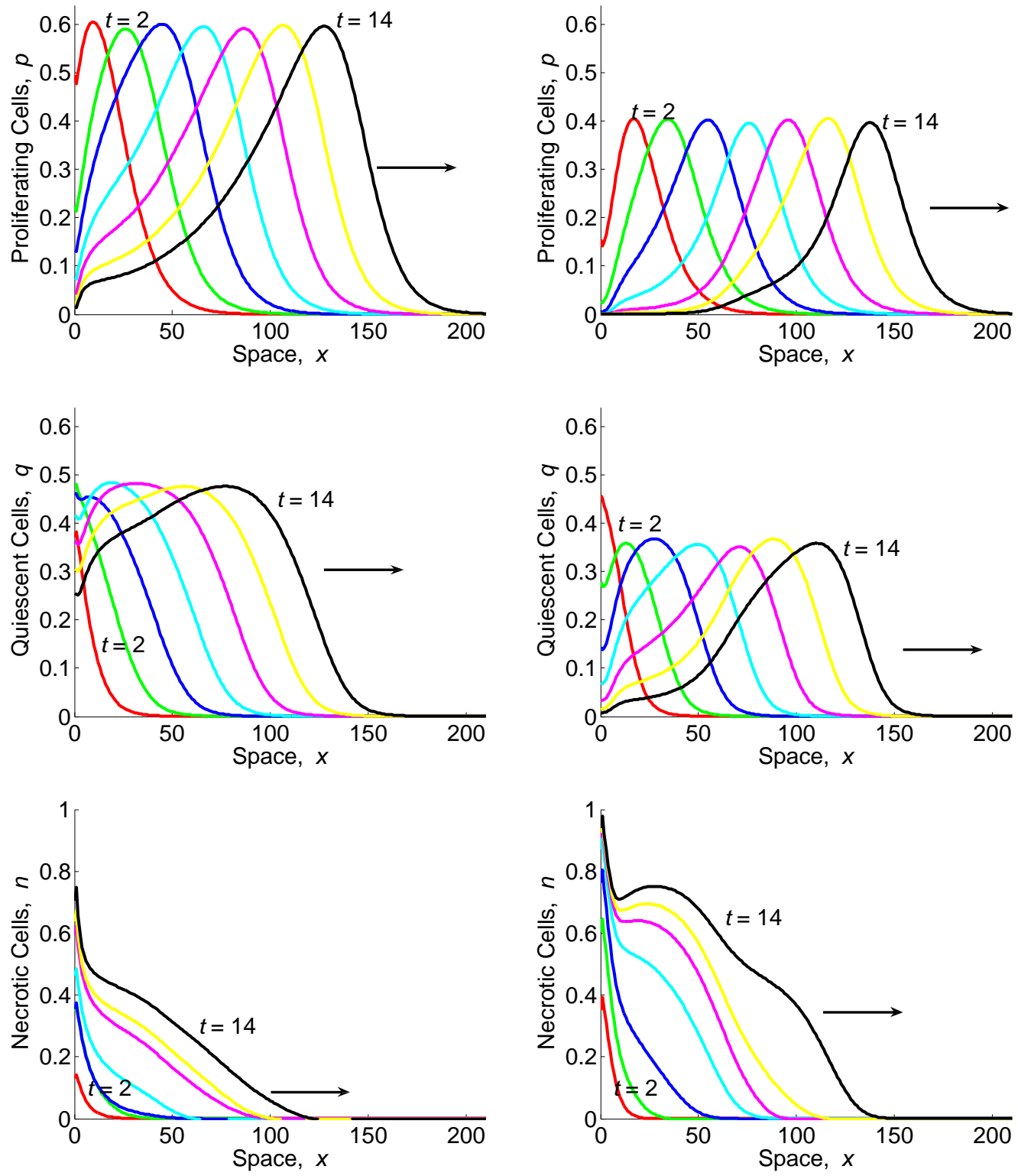

Figure 3: Evolution of proliferat- FigurE 4: Evolution of proliferating, quiescent and necrotic cells at $t=0,2, \ldots, 14$, for $\alpha=0.4$. ing, quiescent and necrotic cells at $\mathrm{t}=0,2, \ldots, 14$, for $\alpha=0.8$. 
proliferating and quiescent cells are still propagating. These results compare well with those obtained by Nirmala et al. [10], whose experimental studies reported no limiting spheroid volume. Instead, growth of the total volume of the spheroid over time was observed.

Tumour cell distributions also vary with nutrient concentration $\alpha$. At a lower $\alpha$ value of 0.4 , and hence increased access of nutrients, live tumour cell distribution is built to a higher level more quickly over a longer span of space (Figure 3). The layer of live tumour cells thickens and the necrotic core diminishes in size. These results are consistent with the argument that tumour growth is influenced not only by the availability of nutrients, but also by the stochastically perturbed tumour environment.

With a lower value of $\alpha$, part of the proliferating cell density at $t=14$ is being distributed in the necrotic core. As compared to the random effects considered in the cell proliferation rate and the nutrient level in the model proposed by Tan and Ang [12], the stochastic perturbation in the present model assumes the form of a white noise process incorporated in the necrotic cell growth dynamics. The observed response of the actively proliferating cells against the necrotic core pressure suggests that tumour growth dynamics could likely be impacted by the stochastic perturbation.

By assuming radial symmetry, we may suppose that the cells move radially so that there is only one nonzero velocity component, which depends only on the distance from the spheroid centre, $r$, and time. Using this assumption and results from the model, it is possible to create a simulation of tumour growth in two dimensions, to provide a more suitable form of visualisation. In Figure 5, a series of snapshots of tumour growth (with $\alpha=0.8$ ) generated in this manner that corresponds to its one dimensional plot is presented.

At $t=2$ the modelled tumour has a higher concentration of proliferating than quiescent and necrotic cells. At $t=6$ there are not only cells moving towards the periphery but a significant number of quiescent and proliferating tumour cells begin to move towards the core area. This simulated internal- 

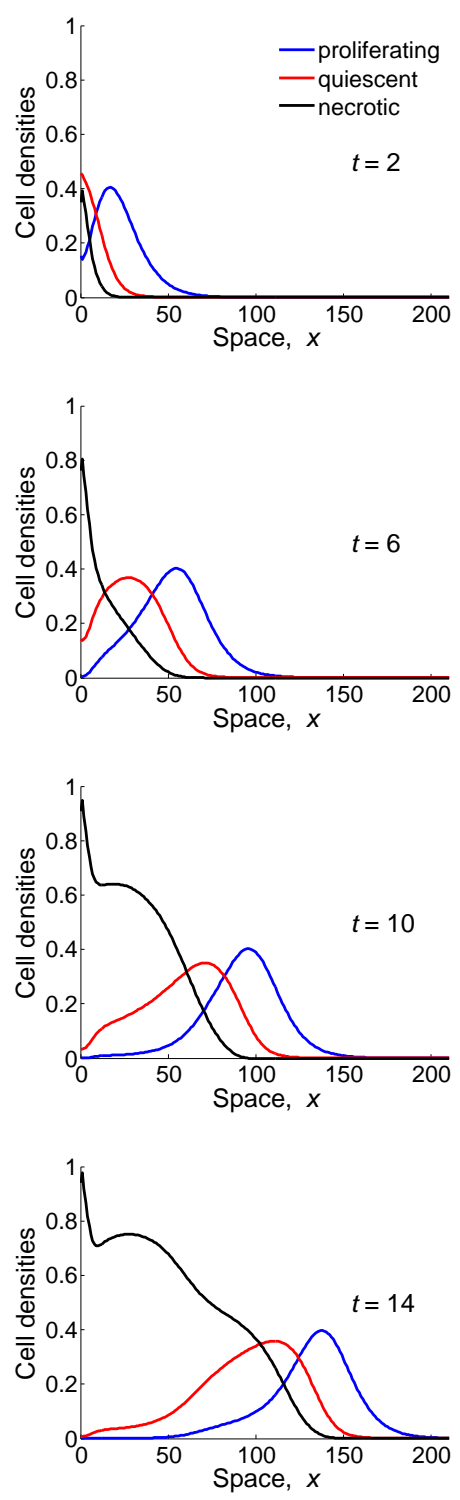

FiguRE 5: Spatial-temporal evolution of avascular tumour cells with white noise. Graphs and snapshots of growing tumour at $t=2,6,10$ and 14 for $\alpha=0.8$ show formation of proliferating, quiescent and necrotic cells. 
ization of tumour cells is also observed in the experimental work by Dorie et al. [5].

From $t=4$ to $t=8$ the tumour is observed to be growing very fast in the initial nutrient rich condition. As the tumour grows in size, the availability of nutrients decreases towards the centre, with growth retardation observed from $t=10$ to $t=12$. This observed growth rate is consistent with experimental works on diffusion-limited tumour growth [7].

At $t=14$ the three layer structure of multicellular spheroid experimentally observed by Folkman and Hochberg [6] and represented in Figure 1 is formed with a distinct necrotic core. The figure shows that the present model has produced fairly acceptable results which are in good agreement with experimental works.

\section{Conclusion}

This article discussed a tumour growth model that includes white noise. Numerical results and qualitative comparisons with published works indicate that the inclusion of a stochastic component results in a more realistic simulation of avascular tumour evolution.

However, the tumour dynamics responsible for the stochastic perturbation has not been determined experimentally. The results presented only speculate about the true nature of the tumour cell distribution based on the assumptions of the model. Further experimental input is required to determine the actual phenomena contributing to stochastic processes in tumour growth. Nonetheless, the model described in this article provides a foundation needed for future studies in tumour growth models with white noise. 


\section{References}

[1] Abercrombie, M., Contact inhibition in tissue culture, In vitro., 6 , 1970, 128-140. doi:10.1007/BF02616114 C573

[2] Adam, J. A., A simplified mathematical model of tumour growth, Mathematical Biosciences, 81, 1986, 224-229. doi:10.1016/0025-5564(86)90119-7 C570

[3] Albano, G. and Giorno, V., A stochastic model in tumor growth, Journal of Theoretical Biology, 242, 2006, 329-336. doi:10.1016/j.jtbi.2006.03.001 C570

[4] Burton, A. C., Rate of growth of solid tumours as a problem of diffusion, Growth, 30, 1966, 157-176. C570

[5] Dorie, M., Kallman, R. and Coyne, M., Effect of cytochalasin b, nocodazole and irradiation on migration and internalization of cells and microspheres in tumor cell spheroids, Experimental Cell Research, 166, 1986, 370-378. doi:10.1016/0014-4827(86)90483-0 C580

[6] Folkman, J., and Hochberg, M., Self-regulation of growth in three dimensions, Journal of Experimental Medicine, 138, 1973, 745-753. C580

[7] Folkman, J., Tumour Angiogenesis, Advances in Cancer Research, 43, 1985, 175-203. C580

[8] Higham, D. J., An algorithmic introduction to numerical simulation of stochastic differential equations, SIAM Review, 43, 2001, 525-546. doi:10.1137/S0036144500378302 C574

[9] Lo, C. F., Stochastic Gompertz model of tumour cell growth, Journal of Theoretical Biology, 248, 2007, 317-321.

doi:10.1016/j.jtbi.2007.04.024 C570 
[10] Nirmala, C., Rao, J. S., Ruifrok, A. C., Langford, L. A. and Obeyesekere, M., Growth characteristics of glioblastoma spheroids, International Journal of Oncology, 19, 2001, 1109-1115. C578

[11] Sheratt, J. A. and Chaplain, M. A. J., A new mathematical model for avascular tumour growth, Journal of Mathematical Biology, 43, 2001, 291-312. doi:10.1007/s002850100088 C571, C572, C575

[12] Tan, L. S. and Ang, K. C., A numerical simulation of avascular tumour growth, ANZIAM Journal, 46(E), 2005, C902-C917.

http://anziamj. austms.org.au/ojs/index.php/ANZIAMJ/article/ view/997 C571, C574, C575, C578

[13] Ward, J. P., and King, J. R., Mathematical modelling of avascular tumour-growth, IMA Journal of Mathematics Applied in Medicine and Biology, 14, 1997, 39-69. doi:10.1093/imammb/14.1.39 C570

\section{Author addresses}

1. Keng-Cheng Ang, Mathematics and Mathematics Education, Nanyang Technological University, 1 Nanyang Walk, Singapore. mailto:kengcheng.ang@nie.edu.sg

2. Liang-Soon Tan, Ministry of Education, SingaPore. mailto:tan_liang_soon@moe.gov.sg 